[Aus dem hygienischen Institut der Universität Berlin.]

\title{
Die Widerstandsfähigkeit der Cholerabacterien gegen das Eintrocknen und gegen Hitze.
}

\author{
Von
}

Dr. med. S. Kitasato

aus Tokio.

Wie bekannt, hat Koch schon in der ersten Choleraconferenz ${ }^{1}$ mitgetheilt, dass die Cholerabacterien beim Eintrocknen sehr bald zu Grunde gehen. Er hat bacillenhaltige Pepton-Fleischbrühe auf dem Deckglas eintrocknen lassen, um zu sehen, wie lange die Cholerabacterien lebensfähig bleiben würden. Dabei stellte sich heraus, dass die Cholerabacterien, nachdem sie eine viertel, eine halbe und eine ganze Stunde getrocknet waren, noch zur Entwickelung kamen, aber manchmal schon nach zwei Stunden abgestorben waren; äber drei Stunden konnte er bei diesen Versuchen die Bacillen nicht am Leben erhalten. Er sagte dabei ausdrücklich: Nur wenn compacte Massen von Bacillenculturen, z. B. die breiartige Substanz einer auf Kartoffeln gewachsenen Cultur getrocknet wurden, hielten sich die Bacillen etwas länger lebensfähig; offenbar weil in diesem Falle sehr viel später ein vollständiges Eintrocknen erfolgte. Aber auch unter diesen Verhältnissen ist es ihm nie gelungen, die Bacillen länger als 24 Stunden in trockenem Zustande lebensfähig zu erhalten.

Dieses Resultat beweist schon, dass die Cholerabacterien unter den gegebenen Bedingungen keinen Dauerzustand haben, welcher ihnen eine gewisse Widerstandsfähigkeit hätte verleihen müssen.

Nachher kamen Andere, welche einen Dauerzustand der Cholera-

${ }^{1}$ Conferenz zur Erörterung der Cholerafrage. Berliner klinische Wochenschrift. 1884. Nr. 31. 
bacterien gefunden haben wollen. So haben Carillon ${ }^{1}$ und Ferra ${ }^{2}$ die kugligen Auftreibungen, $\mathrm{Ceci}^{3}$ die Bildung nicht färbbarer Partieen als Fructificationsvorgang angesprochen. Dann hat Hüppe eine Dauerform der Cholerabacterien beschrieben, auch van Ermengem ${ }^{5}$ hielt die Bildung einer Dauerform für wahrscheinlich. Die Berechtigung zu der Annahme, dass es sich wirklich um eine Dauerform handelt, würde erst vorliegen, wenn bestimmt nachgewiesen wäre, dass die betreffenden Gebilde wesentlich resistenter und zur Erhaltung der Art namentlich dadurch geeignet sind, dass sie das Eintrocknen und Hitze besser ertragen als die gewöhnlichen Cholerabacillen. Derartige Eigenschaften sind aber weder für die Hüppe'schen Kügelchen, noch für die Dauerformen der vorgenannten Autoren nachgewiesen. Hüppe giebt zwar kurz an, dass die von ihm gesehenen Gebilde gegen das Eintrocknen wohl in Folge ihrer stärkeren Gallerthülle resistenter als die vegetativen, kommaähnlichen Zellen seien; aber bei der grossen Wichtigkeit einer solchen Thatsache hätte die Behauptung entschieden mit ausführlichen Beweisen belegt werden müssen.

Um über diese wenig bestimmten und theilweise sich widersprechenden Angaben eine Aufklärung zu schaffen, habe ich im hiesigen Institute die Widerstandsfähigkeit der Cholerabacterien, welche in verschiedener Weise gezüchtet waren, gegen das Eintrocknen und gegen Hitze geprüft. Die Resultate meiner Untersuchungen habe ich im Nachfolgenden zulsammengefasst.

Zuerst habe ich verschieden alte Choleraculturen und zwar von verschiedenen Nährsubstraten untersucht, um zu erfahren, ob zwischen den älteren und jüngeren Choleraculturen und ebenso zwischen den auf ver- schiedenen Nährsubstraten gewachsenen irgend ein Unterschied in Bezug auf Widerstandsfähigkeit gegen Eintrocknen und Hitze zu finden wäre. Mit diesen Culturen habe ich theils Seidenfäden getränkt, theils habe ich sie auf Deckgläser gestrichen, und von beiden Proben eine Abtheilung im Exsiccator über Schwefelsäure, eine zweite Abtheilung im sterilisirten Doppelschälchen an der Luft und eine dritte zur Controle in der feuchten Kammer, d. h. in einer mit angefeuchtetem Fliesspapier ausgekleideten Doppelschale über Wasser aufbewahrt; alle diese Proben befanden sich bei Zimmertemperatur von 20 bis $22^{\circ} \mathrm{C}$. Von Stunde zu Stunde wurden Deckgläser und Seidenfäden entnommen und jedes Mal sowohl in Bouillon

\footnotetext{
1 Semaine médicale. 1884. November.

2 Gazetta medica. Catalana. 1885. Jan.

${ }^{3}$ Semaine médicale. 1885. März.

4 Fortschritte der Medicin. 1885. Nr. 19.

5 Deutsche medicinische Wochenschrift. 1885. Nr. 29.
} 
Widerstandsfähigleit der

\begin{tabular}{|c|c|c|c|c|c|c|c|c|c|c|c|c|c|c|c|}
\hline \multirow{3}{*}{\multicolumn{2}{|c|}{ Zeit }} & \multicolumn{2}{|c|}{$I$} & \multicolumn{2}{|c|}{ II } & \multicolumn{2}{|c|}{ III } & \multicolumn{2}{|c|}{ IV } & \multicolumn{2}{|c|}{$\mathrm{V}$} & \multicolumn{2}{|c|}{ VI } & \multicolumn{2}{|c|}{ VII } \\
\hline & & \multicolumn{2}{|c|}{$\begin{array}{l}\text { Agarcultur } \\
\text { 14 Monate ait } \\
\text { (10 Tage im } \\
\text { Brütofen) }\end{array}$} & \multicolumn{2}{|c|}{$\begin{array}{c}\text { Agarcuitur } \\
60 \text { Tage alt } \\
\text { (12 Tage im } \\
\text { Brütufen) }\end{array}$} & \multicolumn{2}{|c|}{$\begin{array}{c}\text { Agarcultur } \\
9 \text { Tage alt } \\
\text { (1 Tag im } \\
\text { Brütofen) }\end{array}$} & \multicolumn{2}{|c|}{$\begin{array}{l}\text { Agarcultur } \\
9 \text { Tage alt } \\
\text { (nur Zimmer- } \\
\text { tomperatur) }\end{array}$} & \multicolumn{2}{|c|}{$\begin{array}{c}\text { Agarcultur } \\
1 \text { Tag alt } \\
\text { (1 Tag im } \\
\text { Brütofen) }\end{array}$} & \multicolumn{2}{|c|}{$\begin{array}{c}\text { Kartoffel- } \\
\text { cultur } \\
17 \text { Tage alt } \\
\text { (8 Tage im } \\
\text { Brütofen) } \\
\end{array}$} & \multicolumn{2}{|c|}{$\begin{array}{l}\text { Kartoffel- } \\
\text { cultur } \\
\text { 10 Tage alt } \\
\text { (10 Tage im } \\
\text { Brütofen) }\end{array}$} \\
\hline & & $a$ & $b$ & $a$ & $b$ & $a$ & $b$ & $a$ & $b$ & $a$ & 6 & $a$ & $\bar{b}$ & $a$ & $b$ \\
\hline & Stund. $\left\{\begin{array}{l}1 \\
2\end{array}\right.$ & \pm & $\ddagger$ & \pm & \pm & \pm & \pm & \pm & \pm & \pm & \pm & \pm & \pm & \pm & + \\
\hline 2 & , $\left\{\begin{array}{l}1 \\
2\end{array}\right.$ & 7 & + & + & \pm & \pm & + & + & + & + & \pm & + & \pm & + & \pm \\
\hline 3 & $" \quad\left\{\begin{array}{l}1 \\
2\end{array}\right.$ & \pm & $\stackrel{+}{+}$ & + & \pm & $t$ & \pm & 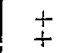 & $\ddagger$ & + & + & + & \pm & $\dot{t}$ & \pm \\
\hline 4 & $\because \quad\left\{\begin{array}{l}1 \\
2\end{array}\right.$ & \pm & + & \pm & \pm & \pm & \pm & + & $\ddagger$ & + & + & + & + & + & + \\
\hline 5 & $\Rightarrow \quad\left\{\begin{array}{l}1 \\
2\end{array}\right.$ & $\dot{t}$ & + & \pm & + & + & $\ddagger$ & $\ddagger$ & + & $t$ & + & + & + & + & + \\
\hline 6 & $\Rightarrow \quad\left\{\begin{array}{l}1 \\
2\end{array}\right.$ & \pm & + & \pm & \pm & + & $\ddagger$ & + & \pm & $t$ & \pm & $t$ & + & + & \pm \\
\hline 7 & $\Rightarrow \quad\left\{\begin{array}{l}1 \\
2\end{array}\right.$ & + & + & \pm & \pm & \pm & \pm & + & \pm & + & + & $t$ & + & + & + \\
\hline 8 & $״ \quad\left\{\begin{array}{l}1 \\
3\end{array}\right.$ & $\ddagger$ & $\ddagger$ & \pm & $\mp$ & \pm & + & + & \pm & + & + & $t$ & + & $\ddagger$ & + \\
\hline 9 & $\Rightarrow \quad\left\{\begin{array}{l}1 \\
2\end{array}\right.$ & $t$ & \pm & $\ddagger$ & \pm & $\ddagger$ & + & \pm & \pm & $t$ & + & $\ddagger$ & + & + & + \\
\hline 10 & $\Rightarrow \quad\left\{\begin{array}{l}1 \\
3\end{array}\right.$ & \pm & + & \pm & \pm & $t$ & \pm & + & $t$ & + & $t$ & \pm & + & $t$ & \pm \\
\hline 15 & " $\quad\left\{\begin{array}{l}1 \\
2\end{array}\right.$ & + & + & \pm & \pm & $\ddagger$ & + & \pm & \pm & \pm & + & \pm & + & + & $\stackrel{t}{t}$ \\
\hline $2 \pm$ & $״ \quad\left\{\begin{array}{l}1 \\
2\end{array}\right.$ & \pm & + & $t$ & + & + & + & $t$ & $t$ & $t$ & $t$ & $I$ & + & $t$ & $t$ \\
\hline 30 & $\because \quad\left\{\begin{array}{l}1 \\
2\end{array}\right.$ & + & \pm & \pm & $\dot{+}$ & $t$ & + & + & 8 & + & $t$ & \pm & 吉 & \pm & 음 \\
\hline 40 & $" \quad\left\{\begin{array}{l}1 \\
2\end{array}\right.$ & \pm & + & + & \pm & + & $\vec{\square}$ & + & $=$ & $t$ & $t$ & $t$ & $\underline{9}$ & \pm & $\bar{z}$ \\
\hline & l'age $\left\{\begin{array}{l}1 \\
2\end{array}\right.$ & $t$ & 뭉 & $\stackrel{+}{+}$ & 음 & + & $=$ & \pm & 二 & $t$ & 8 & q & $=$ & $t$ & $=$ \\
\hline 3 & $\Rightarrow \quad\left\{\begin{array}{l}1 \\
2\end{array}\right.$ & \pm & $=$ & \pm & $=$ & + & $=$ & $t$ & $=$ & + & $=$ & $\mp$ & $=$ & 9 & $\bar{z}$ \\
\hline 4 & $\Rightarrow \quad\left\{\begin{array}{l}1 \\
2\end{array}\right.$ & 9 & $=$ & 웅 & $=$ & 은 & = & 9 & $\bar{z}$ & $\bar{t}$ & $=$ & I & $=$ & $\mp$ & $\overline{-}$ \\
\hline 5 & $\Rightarrow \quad\left\{\begin{array}{l}1 \\
2\end{array}\right.$ & $\bar{t}$ & $=$ & $\mp$ & $=$ & $\mp$ & $=$ & $\vec{t}$ & $\overline{-}$ & $\mp$ & $=$ & $\bar{\square}$ & $=$ & $\mp$ & $\underline{-}$ \\
\hline 6 & $\Rightarrow \quad\left\{\begin{array}{l}1 \\
2\end{array}\right.$ & $\mp$ & $=$ & $\bar{t}$ & $=$ & $\mp$ & $\bar{z}$ & $\mp$ & $=$ & $\mp$ & $=$ & $=$ & $=$ & $\bar{\square}$ & $\bar{z}$ \\
\hline 7 & $" \quad\left\{\begin{array}{l}1 \\
2\end{array}\right.$ & $\bar{\square}$ & 二 & $\mp$ & $=$ & $\mp$ & $=$ & $\mp$ & $\bar{z}$ & $\vec{t}$ & $=$ & $=$ & $=$ & $=$ & $=$ \\
\hline 8 &,$\quad\left\{\begin{array}{l}1 \\
2\end{array}\right.$ & $=$ & $=$ & $\mp$ & $=$ & $\mp$ & $=$ & $\mp$ & $=$ & $\mp$ & $=$ & $\overline{-}$ & $=$ & $=$ & $=$ \\
\hline 9 & $=\left\{\frac{1}{3}\right.$ & $=$ & $=$ & $\mp$ & $=$ & $\bar{\square}$ & $=$ & $\mp$ & $\bar{z}$ & $\bar{t}$ & $=$ & $\bar{z}$ & $\overline{-}$ & $=$ & $\bar{z}$ \\
\hline 10 & $\Rightarrow \quad\left\{\begin{array}{l}1 \\
3\end{array}\right.$ & $=$ & $\bar{z}$ & $\mp$ & $=$ & $\overline{-}$ & $=$ & \pm & $=$ & $\bar{t}$ & $\bar{z}$ & $=$ & $=$ & $=$ & $\vec{z}$ \\
\hline 11 & $" \quad\left\{\begin{array}{l}1 \\
2\end{array}\right.$ & $\bar{z}$ & $\bar{z}$ & $\bar{\square}$ & $=$ & $=$ & $=$ & $\mp$ & $=$ & $\vec{\square}$ & 二 & $=$ & $=$ & $=$ & $=$ \\
\hline 12 & $\Rightarrow \quad\left\{\begin{array}{l}1 \\
2\end{array}\right.$ & $\bar{z}$ & $\vec{z}$ & $=$ & $=$ & $=$ & $=$ & $\mp$ & - & $=$ & $=$ & $=$ & $=$ & $=$ & $=$ \\
\hline 13 & $\Rightarrow \quad\left\{\frac{1}{2}\right.$ & $=$ & $=$ & $=$ & $=$ & $=$ & $=$ & $\mp$ & $=$ & $=$ & 亡 & $=$ & - & $=$ & $=$ \\
\hline 14 & $\Rightarrow \quad\left\{\begin{array}{l}1 \\
2\end{array}\right.$ & $=$ & $\bar{z}$ & $=$ & I & $=$ & $=$ & $\bar{\square}$ & $=$ & $=$ & $=$ & $=$ & $=$ & $=$ & $=$ \\
\hline 15 &..$\quad\left\{\frac{1}{2}\right.$ & $\overline{-}$ & $=$ & $=$ & $\bar{z}$ & $=$ & $=$ & 二. & $=$ & $=$ & $=$ & $=$ & $=$ & $=$ & $=$ \\
\hline 16 &..$\left\{\begin{array}{l}1 \\
2\end{array}\right.$ & $=$ & $\bar{z}$ & $=$ & $=$ & $=$ & $=$ & $=$ & $\bar{z}$ & $=$ & $=$ & $=$ & $=$ & $=$ & - \\
\hline 17 & $\because \quad\left\{\begin{array}{l}1 \\
2\end{array}\right.$ & $=$ & $=$ & $=$ & $\bar{z}$ & $=$ & $=$ & $=$ & $=$ & $=$ & $=$ & $=$ & $=$ & $=$ & $=$ \\
\hline 18 & $" \quad\}_{2}^{1}$ & $\bar{z}$ & $\bar{z}$ & $=$ & $=$ & $=$ & $=$ & $\bar{z}$ & $=$ & $=$ & $\bar{z}$ & $\dot{-}$ & $\Xi$ & $=$ & $=$ \\
\hline
\end{tabular}




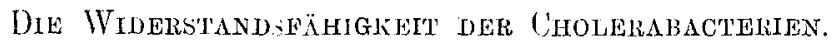

Culturen gegen Eińtrocknen.

\begin{tabular}{|c|c|c|c|c|c|c|c|c|c|c|c|c|c|c|c|}
\hline \multirow{2}{*}{\multicolumn{2}{|c|}{$\begin{array}{c}\text { VIII } \\
\text { Kartoffel- } \\
\text { cultur } \\
\text { 8 Tage alt } \\
\text { (8 Tage im } \\
\text { Britofen) }\end{array}$}} & \multicolumn{2}{|c|}{ IX } & & \multicolumn{2}{|c|}{$X I$} & \multicolumn{2}{|c|}{ XII } & \multicolumn{2}{|c|}{$\mathrm{XIII}$} & \multicolumn{2}{|c|}{ XIV } & \multicolumn{2}{|c|}{$\mathrm{XV}$} \\
\hline & & $\begin{array}{c}\text { Gel } \\
\text { cu } \\
102 \mathrm{~T}\end{array}$ & & $\begin{array}{l}\text { Gel } \\
\mathrm{cu} \\
12 \mathrm{~T}\end{array}$ & & & & $\begin{array}{r}\text { Bou } \\
\mathrm{cu} \\
106 \\
(3 \mathrm{~T} . \mathrm{i} \\
\text { Flo }\end{array}$ & & $\begin{array}{r}\text { Bou } \\
\text { cu } \\
15 \mathrm{~T} \\
2 \mathrm{~T} \\
\text { Huitec } \\
\text { Obe }\end{array}$ & & $\begin{array}{c}\text { Bou } \\
\mathrm{cu} \\
1 \mathrm{~T} \\
\text { (im B } \\
\text { nur F }\end{array}$ & & & \\
\hline$a$ & $b$ & $a$ & $b$ & $\stackrel{a}{a}$ & $b$ & $a$ & $\bar{b}$ & $a$ & $b$ & $a$ & $b$ & $a$ & $b$ & $a$ & $\underline{b}$ \\
\hline \pm & \pm & \pm & \pm & + & + & $t$ & $t$ & + & + & + & + & + & + & $t$ & + \\
\hline$T$ & \pm & + & + & + & & & $\begin{array}{l}+ \\
+\end{array}$ & $\dot{t}$ & $\begin{array}{l}t \\
+\end{array}$ & $t$ & & $\stackrel{t}{t}$ & & & + \\
\hline+ & + & + & + & + & $I$ & + & + & F & $I$ & $I$ & + & $I$ & \pm & + & \\
\hline$\mp$ & + & + & $\stackrel{t}{+}$ & $t$ & $\stackrel{+}{\dagger}$ & + & + & + & 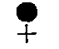 & $\dot{t}$ & \pm & \pm & 음 & + & 9 \\
\hline+ & $\mp$ & $t$ & + & + & $\mp$ & + & + & \pm & $\bar{\square}$ & + & + & + & $=$ & + & $\vec{\square}$ \\
\hline+ & \pm & \pm & \pm & + & + & + & + & \pm & $=$ & \pm & \pm & \pm & $=$ & + & $=$ \\
\hline+ & + & + & \pm & \pm & + & \pm & \pm & \pm & 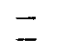 & \pm & \pm & \pm & $=$ & \pm & $=$ \\
\hline+ & + & + & \pm & $t$ & $t$ & $\ddagger$ & + & \pm & $=$ & \pm & + & + & $=$ & $t$ & $=$ \\
\hline+ & + & + & \pm & \pm & \pm & 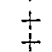 & + & \pm & $\Xi$ & + & \pm & \pm & $=$ & \pm & $\Xi$ \\
\hline+ & $\ddagger$ & \pm & \pm & + & + & $\stackrel{+}{+}$ & \pm & \pm & $=$ & \pm & 움 & \pm & $=$ & \pm & $=$ \\
\hline+ & \pm & $t$ & \pm & \pm & \pm & \pm & \pm & \pm & $\bar{z}$ & \pm & $\bar{z}$ & \pm & $=$ & \pm & $=$ \\
\hline \pm & \pm & \pm & \pm & \pm & + & $t$ & \pm & \pm & $=$ & \pm & $=$ & $t$ & 二 & \pm & $=$ \\
\hline+ & 9 & + & + & + & + & + & + & $t$ & - & + & - & + & - & $t$ & - \\
\hline & & & & & & & & & & & - & & - & $t$ & \\
\hline+ & $\bar{\square}$ & + & 9 & \pm & \pm & + & 9 & $\ddagger$ & $=$ & \pm & $\bar{z}$ & 9 & $\bar{z}$ & + & $=$ \\
\hline \pm & $=$ & + & ח & + & 0 & + & - & + & - & + & - & $\overline{7}$ & - & 욤 & - \\
\hline & & & $\square$ & & & & & & - & + & - & $\square$ & - & $\square$ & - \\
\hline+ & $\vec{z}$ & + & $\bar{z}$ & $\stackrel{t}{+}$ & $\Xi$ & + & $\vec{\square}$ & + & $\overline{-}$ & \pm & $\bar{z}$ & $=$ & $=$ & $\Xi$ & $\overline{-}$ \\
\hline+ & $=$ & $\bar{I}$ & $\bar{z}$ & 웅 & - & \pm & - & $\bar{\square}$ & - & 8 & - & - & - & - & - \\
\hline- & - & & 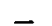 & & & 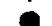 & & & & & $\bar{I}$ & & - & - & \\
\hline+ & - & + & - & - & 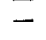 & + & $=$ & - & - & & $\overline{-}$ & $=$ & $=$ & - & $=$ \\
\hline $\overrightarrow{0}$ & $\vec{z}$ & $\bar{\square}$ & $\bar{z}$ & $\bar{z}$ & $\exists$ & $\bar{\square}$ & $=$ & $=$ & $=$ & $z$ & $=$ & $\bar{z}$ & $=$ & $=$ & $=$ \\
\hline- & $=$ & $=$ & $\bar{z}$ & $=$ & J & $=$ & $=$ & $=$ & $=$ & $=$ & $=$ & $=$ & $=$ & $=$ & - \\
\hline 7 & - & & - & - & & 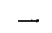 & - & - & _ & - & - & - & - & - & \\
\hline- & & & - & - & & & - & - & - & & - & - & - & - & - \\
\hline 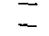 & I & - & $=$ & I & - & $\bar{z}$ & $\overline{-}$ & $=$ & $\bar{z}$ & I & $=$ & $=$ & $=$ & $=$ & - \\
\hline- & - & - & - & & - & - & - & - & - & - & - & - & - & - & - \\
\hline & & & - & & & & 一 & 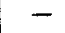 & & & - & - & - & 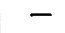 & - \\
\hline 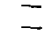 & $z$ & ב & 二 & $z$ & 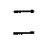 & $I$ & $\bar{z}$ & $\bar{z}$ & $=$ & 二 & $\bar{z}$ & $=$ & Z & $\bar{z}$ & $=$ \\
\hline 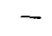 & - & - & - & - & 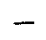 & - & - & - & - & - & - & - & - & - & - \\
\hline & & & & & & & & 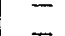 & & & & - & - & - & \\
\hline & & & 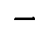 & & & & 二 & - & 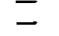 & $\overline{-}$ & $\bar{z}$ & 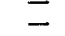 & $=$ & $\bar{z}$ & $=$ \\
\hline 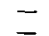 & z & - & - & - & 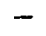 & & - & - & $=$ & - & - & - & 二 & 二 & - \\
\hline & - & & - & & & & & & & & & - & 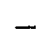 & $\overline{-}$ & \\
\hline & - & - & - & - & & & 二 & - & - & $\bar{z}$ & $=$ & $\overline{-}$ & $=$ & 二 & $\bar{z}$ \\
\hline$z$ & 7 & $=$ & - & - & - & 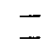 & - & - & - & - & - & - & - & - & - \\
\hline 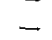 & 7 & 7 & - & & & & $I$ & - & & - & 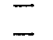 & $\overline{-}$ & $\overline{-}$ & $\overline{-}$ & $I$ \\
\hline- & - & & - & & - & & - & 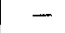 & - & - & - & - & - & - & - \\
\hline$=$ & $=$ & ב & $=$ & $=$ & - & $\bar{z}$ & $=$ & - & $=$ & - & $=$ & - & $=$ & - & - \\
\hline . & - & - & - & - & - & - & - & - & - & - & - & - & - & - & - \\
\hline & - & - & - & - & - & - & - & - & 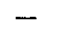 & - & - & $\overline{-}$ & $=$ & $=$ & $=$ \\
\hline
\end{tabular}


Widerstandsfähigkeit der Culturen gegen Hitze.

\begin{tabular}{|c|c|c|c|c|c|c|c|c|}
\hline & I & III & VI & VIII & IX & $\mathrm{x}$ & XII & XIV \\
\hline $50^{\circ} \mathrm{C}$ & & & & & & & & \\
\hline 5 Minuten & + & + & + & + & + & + & + & + . \\
\hline $\begin{array}{l}10 \quad, \\
15\end{array}$ & + & + & + & + & \pm & $\begin{array}{l}+ \\
+\end{array}$ & \pm & \pm \\
\hline $55^{\circ} \mathrm{C}$ & & & & & & & & \\
\hline 5 Minuten & + & + & + & + & + & + & + & + \\
\hline $\begin{array}{l}10 \quad " \\
15 \quad "\end{array}$ & - & + & $t$ & \pm & - & $\stackrel{+}{-}$ & - & - \\
\hline $60^{\circ} \mathrm{C}$ & & & & & & & & \\
\hline 5 Minuten & - & \pm & \pm & $\overline{-}$ & - & 一 & $=$ & - \\
\hline $65^{\circ} \mathrm{C}$ & & & & & & & & \\
\hline 5 Minuten & - & - & - & - & - & - & - & - \\
\hline
\end{tabular}

als auch in Nährgelatine gebracht; die in Bouillon geimpften Proben wurden in den Brütofen bei $36^{\circ} \mathrm{C}$. gestellt, während die in Gelatine gezüchteten nach der Esmarch'schen Rollmethode behandelt und bei Zimmertemperatur von 20 bis $22^{\circ} \mathrm{C}$. aufbewahrt wurden.

Bei den Hitze-Versuchen habe ich zuerst eine Platinöse voll von der Cultur genommen, sie in vorher verflüssigte frische Nährgelatine und diese dann in das Wasserbad gebracht, welches auf die bestimmte Temperatur vorher erwärmt war; diese habe ich dann gerollt.

$\mathrm{Zu}$ bemerken ist noch, dass mehrere Reihen solcher Versuche wiederholt gemacht sind. Das Resultat ist in vorstehenden Tabellen zusammengefasst.

Aus diesen Resultaten lassen sich folgende Schlüsse ziehen:

1. Zwischen älteren und jüngeren Culturen der Cholerabacterien findet sich kein Unterschied bezüglich ihrer Widerstandsfähigkeit gegen Eintrocknen und Hitze.

2. Die Zeitdauer des Absterbens der Cholerabacterien nach dem Eintrocknen hängt von der Art und Weise ab, wie man das Material präparirt. So sind diejenigen Culturen, welche von Seidenfäden aufgenommen sind, verhältnissmässig länger lebensfähig, als diejenigen, welche auf dem Deckglas in dünner Schicht ausgebreitet waren, wenn sie auch ursprünglich aus derselben Cultur genommen waren. Es liegt das offenbar daran, dass bei den ersteren viel später ein vollständiges Eintrocknen erfolgt, als bei den letzteren.

3. Die Zeitdauer des Ábsterbens hängt ferner ab ron der Beschaffenheit der Culturen selbst. So bleiben die Häutchen von der Oberfläche der Bouilloncultur, ebenso wie die breiartige und zäh- 
schleimige Cultur von der Oberfläche des Agar und der Kartoffeln auf dem Deckglas länger lebensfähig als die bacillenhaltige aber flüssige Bouillon, denn die von ersteren auf dem Deckglas gebildete Sehicht ist nach dem Verdunsten der Flüssigkeit viel dicker als die von der flüssigen Bouillon, zurückbleibende. Ausserdem sind diejenigen Proben, welche im Exsiccator über Schwefelsäure aufbewahrt wurden, viel länger widerstandsfähig, als diejenigen, welche im Doppelschälchen an der Luft standen; es erklärt sich dies wohl daraus, dass in diesem Falle die oberflächlichen Schichten der Seidenfäden schneller und intensiver austrocknen and dadureh die inneren Theile derselben lünger einen gewissen Grad von Feuchtigkeit behalten.

4. Ein wesentlich verschiedenes Verhalten der Choleraculturen gegen Temperaturen von 50 bis $60^{\circ}$ hat sich nicht ergeben.

5. Die unter einander abweichenden früheren Angaben über die Widerstandsfähigkeit der Cholerabacterien gegen das Eintrocknen finden in ungezwungener Weise ihre Erklärung in der verschiedenen Art und Weise, unter wclcher das Eintrocknen vor sich geht; je schneller und vollkommener dasselbe erfolgt, um so schneller sterben die Cholerabacterien ab. Einen besonderen Dauerzustand dagegen, welcher die Bacillen an und für sich widerstandsfähiger gegen das Fintrocknen macht, habe ich bei meinen Versuchen nicht nachweisen können.

Hier möchte ich noch bemerken, dass ich bei den älteren Choleraculturen stets die aus dem Zerfall der Cholerabacillen hervorgehenden Kügelchen neben den an Zahl verhältnissmässig sehr geringen Bacillen massenhaft unter dem Mikroskope beobachtet habe. Ich habe nun versucht, mit grösster Sorgfalt nur die Körnchen in andere frische Nährsubstrate (Agar, Gelatine und Bouillon) isolirt zu übertragen; davon habe ich dann eine Platinöse voll auf ein vorher sterilisirtes Deckglas gebracht and es in der von Hüppe empfohlenen Weise auf dem hohlen Objectträger luftdicht befestigt. Derartig hergestellte Präparate habe ich mit Hülfe des von Zeiss construirten heizbaren Mikroskopes mit grösster Genauigkeit und vielfach wiederholt auf das Auskeimen der Körnchen untersucht; leider ist es mir aber in keinem Falle gelungen, das Auswachsen der Körnchen zu Cholerabacillen zu constatiren.

Ferner habe ich eine Bouilloncultur der Cholerabacterien nach 100 Tagen mikroskopisch untersucht und massenhafte Körnchen, dabei aber auch noch Bacillen beobachtet; von dieser Cultur habe ich eine 
Platinöse voll in frische alkalische Nährgelatine gebracht und auf Platten gegossen; nach einigen Tagen wuchsen die Choleracolonieen sowohl auf Original- als auch auf Verdünnungsplatten in reichlicher Zahl. Diesen Versuch habe ich von Zeit zu Zeit mit derselben Cultur wiederholt und dabei gefunden, dass nach 165 Tagen auf der Originalplatte nur noch 21 Colonieen, auf der ersten Verdünnung 5 und auf der zweiten keine Colonie mehr gewachsen sind; bei den mikroskopischen Untersuchungen konnte ich dementsprechend zwischen zahllosen Körnchen nur noch einige Bacillen finden. Nach 180 Tagen war aber auf der Originalplatte nur. eine einzige Choleracolonie gewachsen, während die Verdünnungsplatten ganz steril blieben. Die mikroskopischen Untersuchungen ergaben dabei keinen Bacillus mehr, wohl aber massenhafte Körnchen. Nach 190 und wiederum nach 200 Tagen sind die Versuche wiederbolt gemacht, dabei hat es jedes Mal negative Resultate ergeben.

Daraus folgt, dass die Körnchen in den Choleraculturen zu dem Auskeimen der Bacillen in keiner Beziehung stehen.

\section{Erklärung der Tabellen.}

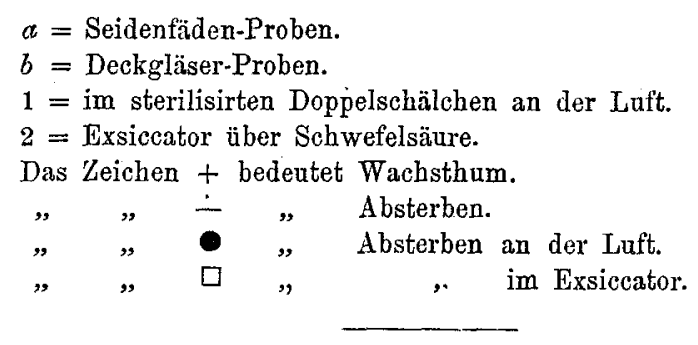

\section{Bemerkungen.}

1. Wenn auch nur eine einzige Choleracolonie gewachsen war, so habe ich dies mit + bezeichnet.

2. Die beiden Resultate der in Bouillon und Gelatine gebrachten Proben sind fast gleich, deshalb habe ich es in der Tabelle einfach zusammengefasst.

3. Bei den Controlversuchen mit denjenigen Proben, welche in der feuchten Kammer aufbewahrt wurden, war das Wachsthum ohne Ausnahme sehr üppig; es trat selbst nach 125 Tagen noch kein Absterben ein. Ieh habe deshalb das positive Resultat der Controlversuche absichtlich aus der Tabelle weggelassen, um die Uebersicht der Tabelle zu vereinfachen. Darüber, wie lange die Cholerabacterien in feuchter Kammer lebensfähig bleiben können, setze ich die Versuche noch weiter fort. 